
\title{
25 Research Soure \\ Risk Factors of Early Periprosthetic Femoral Fracture After Total Knee Arthroplasty
}

Chaturong Pornrattanamaneewong

Siriraj Hospital

Akraporn Sitthitheerarut

Prajuabkirikhan Hospital

Pakpoom Ruangsomboon

Siriraj Hospital

Keerati Chareancholvanich

Siriraj Hospital

Rapeepat Narkbunnam ( $\sim$ mai_parma@hotmail.com )

Siriraj Hospital

\section{Research Article}

Keywords: Risk factor, periprosthetic fracture, total knee arthroplasty, dyslipidemia

Posted Date: May 22nd, 2021

DOI: https://doi.org/10.21203/rs.3.rs-471819/v1

License: (c) (1) This work is licensed under a Creative Commons Attribution 4.0 International License. Read Full License

Version of Record: A version of this preprint was published at BMC Musculoskeletal Disorders on December 1st, 2021. See the published version at https://doi.org/10.1186/s12891-021-04875-5. 


\section{Abstract}

\section{Background}

Periprosthetic femoral fracture (PFF) is a serious complication after total knee arthroplasty (TKA). However, the risk factors of PFF in the early postoperative setting are not well documented. This study determines the risk factors of early PFF after primary TKA.

\section{Methods}

This study recruited 24 patients who had early PFF within postoperative 3 months and 96 control patients. Demographic data (age, gender, weight, height, body mass index, Deyo-Charlson comorbidity index, diagnosis, operated side, underlying diseases and history of steroid usage intraoperative outcomes), intraoperative outcomes (operative time, surgical approach, type and brand of the prosthesis), and radiographic outcomes (distal femoral width; DFW, prosthesis-distal femoral width ratio; PDFW ratio, anatomical lateral distal femoral angle; LDFA, femoral component flexion angle; FCFA and anterior femoral notching; AFN) were recorded and compared between groups. Details of PFF, including fracture pattern, preoperative deformity, and time to PFF were also documented.

\section{Results}

In univariate analysis, the PFF group had significantly older, right side injury, rheumatoid, dyslipidemia, Parkinson patients than the control group $(p<0.05)$. No cruciate-retaining design was used in PFF group $(p=0.004)$. Differences between the prosthetic brand used were found in this study $(p=0.046)$. For radiographic outcomes, PFF group had significantly lower DFW but higher PDFW ratio and LDFA than the control group $(p<0.05)$. While FCFA and AFN were similar between groups. The fracture patterns were medial condylar (45.8\%), lateral condylar $(25.0 \%)$ and supracondylar fracture $(29.2 \%)$. The mean overall time to PFF was $37.2 \pm 20.6$ days (range $8-87$ days). Preoperative deformity was significantly different among the three patterns $(p<0.05)$. When analyzed using the logistic regression model, age and dyslipidemia were only two independent risk factors for early PFF. The cut-off point of age was $>=75$ years, with a sensitivity of $75.0 \%$ and specificity of $78.1 \%$. The odds ratio of dyslipidemia was 6.63 (95\% confidence interval, 1.11 to 39.8 ).

\section{Conclusion}

This study determined that age and dyslipidemia were the independent risk factors for early PFF. However, further well-controlled studies with a larger sample size were needed to address this issue.

\section{Introduction}

Periprosthetic femoral fracture (PFF) is one of the serious complications after total knee arthroplasty (TKA). It is associated with significant morbidity, which requiring further procedures and additional cost[1]. The incidence of PFF has been reported ranging from 0.3 to $2.5 \%$ and typically located in the 
supracondylar region[2,3]. Most of PFF occurs following a low energy trauma such as a simple fall[4]. However, it can also occur in patients without a history of trauma[3].

Several risk factors have been recognized, which can be arranged into the patient, the surgical, and implant-related factors. Patient factors include advanced age, female gender, rheumatoid arthritis (RA), neurologic diseases, osteoporosis, and chronic steroid use[3,5,6]. While some surgical or technical errors such as anterior femoral notching (AFN) may increase the risk of PFF[1]. In terms of implant-related factors, although Alden et al.[7] demonstrated an increased risk of intraoperative femoral fracture with posterior-stabilized(PS) design, this factor is still disputable for postoperative femoral fracture.

Most of the mentioned studies define the risk factors of PFF in the overall postoperative period. The risk factors of PFF in the early postoperative setting are not well reported. Thus, the purpose of this study is to find out the risk factors of early PFF after primary TKA. Our results may help the surgeon to create strategic planning for preventing this devastating complication.

\section{Methods}

The institutional review board approved this study protocol and registered as TCTR20200226001. Between March 2008 and May 2019, the patients who underwent primary TKA in our institute and had PFF within 3 months after surgery were recruited (PFF group). We excluded the patients who had PFF related to high energy trauma. In order to reduce the differences of surgical techniques and instruments used in each time period, the patients who had no PFF within 3 months were selected as the followings; when we met the PFF case, two consecutive patients who underwent before and two consecutive patients who underwent after PFF case were recruited and collected as the control group. The exclusion criteria of both groups were the patients required stem or metal augmentation, intraoperative fracture or consequent intraoperative fracture from immediate postoperative radiograph, collateral ligament injury, periprosthetic joint infection, and incomplete data. All procedures were performed in accordance with relevant guidelines.

Patients' characteristics including age, gender, weight, height, body mass index (BMI), DeyoCharlson comorbidity index (DCCl), diagnosis, operated side, underlying diseases, and history of steroid usage were collected. Operative time, surgical approach, type, and brand of the prosthesis were recorded as the intraoperative outcomes. For postoperative radiographic outcomes, three measurements were made on anteroposterior knee radiographs; the anatomical lateral distal femoral angle (LDFA), distal femoral width (DFW), and prosthesis-distal femoral width (PDFW) ratio. First, the distal femoral joint line (DJL) was drawn using the line connecting the distal-most aspects of medial and lateral condyles of the femoral component. The anatomical femoral axis (AFA) was defined as the line connecting two midpoints of the femoral shaft at 5 and $10 \mathrm{~cm}$ above the DJL. The LDFA was the lateral angle between the AFA and DJL. The DFW was defined as the longest distance of the distal femur that parallels to the DJL. Furthermore, the PDFW ratio was the ratio of the mediolateral width of the femoral component to DFW. The femoral component flexion angle (FCFA) and anterior femoral notching (AFN) were measured 
on the lateral view of the knee radiograph. The sagittal femoral axis (SFA) was drawn using the line connecting two midpoints of the femoral shaft at 5 and $10 \mathrm{~cm}$ above the most distal part of the femoral component. The FCFA was the posterior angle between SFA and the sagittal plane of the femoral component. The AFN was measured as the depth between anterior femoral cortex and the anterior cut line of the distal femur(8) (Figure 1). Details of PFF, including fracture pattern, preoperative deformity, preoperative anatomical femorotibial angle (aFTA), and time to PFF were also recorded.

\section{Statistical analysis}

The statistical analysis was performed using SPSS program version 18.0 (SPSS Inc., Chicago, Illinois). In the univariate analysis, the Student t-test was used to compared continuous data between groups. Consequently, analysis of variance was used to compare continuous data among the different fracture patterns. The Chi-square or Fisher-exact test was used to compared categorical data in our study. The multivariate analysis was then performed to find out the independent risk factors for PFF. All variables with $p$-value $<0.2$ were entered into a logistic regression model. The significant risk factor was considered if a $\mathrm{p}$-value $<0.05$.

\section{Results}

A total of 24 PFF patients were included for analysis. Thus 96 controlled patients were selected in this study. The patients' characteristics of both groups were shown in Table 1. The mean age in the PFF group was significantly higher than the control group $(p<0.001)$. The majority of overall patients were female. PFF group had more right-sided injury than the control group $(p=0.012)$. More RA patients were found in the PFF group $(p=0.039)$. The PFF patients had a significantly higher prevalence of underlying dyslipidemia and Parkinson's disease than the control group ( $p=0.040$ and 0.007 , respectively). The use of cruciate-retaining (CR) design was significantly lower in the PFF group ( $p=0.004)$. A significant difference in the prosthesis brand used was also found between groups $(p=0.046)$. In radiographic outcomes, as shown in Table 2, the PFF group had significantly lower DFW than the control group ( $p=$ 0.001), while the PDFW ratio and LDFA were significantly higher in the PFF group ( $p=0.004$ and 0.026 , respectively). After using logistic regression analysis, age and dyslipidemia were significantly independent risk factors for PFF ( $p=0.003$ and 0.039 , respectively). An odds ratio of dyslipidemia was 6.63 (95\% confidence interval (Cl), 1.11 to 39.8). To identify the cut-off point for age, we performed the post-hoc analysis using the receiver operating characteristics (ROC) curve (Figure 2). The area under the curve was $0.806(95 \% \mathrm{Cl}, 0.700$ to $0.912, \mathrm{p}<0.001)$. Finally, the cut-off point of age $>=75$ years that provided the highest summation of sensitivity and specificity was determined. There were 18 PFF and 21 control patients In the patients aged $>=75$ years. The sensitivity, specificity, positive and negative predictive value were $75.0 \%, 78.1 \%, 46.2 \%$ and $92.6 \%$, respectively.

Table 3 demonstrated the analysis of the pattern of PFF; the most common location was medial condylar fractures (11 cases, $45.8 \%)$. While 7 (29.2\%) and 6 (25.0\%) cases had supracondylar and lateral condylar fractures, respectively. Significant differences of preoperative deformity and aFTA were found among 
three patterns of PFF ( $p=0.036$ and 0.036 , respectively). All lateral condylar fractures were associated with preoperative valgus deformity. Nevertheless, most supracondylar fractures were related to preoperative varus deformity. The mean overall time to PFF was $37.2 \pm 20.6$ days (range $8-87$ days). Although supracondylar fractures had a longer time than the others, these differences did not reach statistical significance $(p=0.266)$.

\section{Discussion}

Our study was the first investigation that ascertained the risk factors of PFF in the early postoperative period within 3 months. In univariate analysis, our significant factors, including age, RA, and neurologic disease, were similar to previous studies[3,5,6]. Although several pre-existing neurological diseases, including epilepsy, Parkinson's disease, and poliomyelitis were proposed as the risk factors[9], our study only had Parkinson patients. These diseases were related to an increase in falls. Regarding the operated site, Zainul-Abidin et al.[1] reported left-sided surgery was a significant risk factor. However, the opposite side was reported in our study. The relevance of this factor was still unexplained.

In terms of prosthetic design, our study revealed no CR design used in PFF group. Besides, we also demonstrated that PS design was the risk factor for early PFF, which this factor had never been reported. Alden et al.[7] reviewed 49 intraoperative femoral fractures from 17,389 primary TKA. These fractures could occur during exposure, bone preparation, and trialing of the component. They found that the PS design had a higher risk of intraoperative femoral fracture than CR design. The relative risk was 4.74. From their conclusions, we hypothesized that the intercondylar box cut of PS design might cause stress riser or intraoperative occult fracture. It might lead to early PFF in some patients.

The relationship between prosthetic and distal femoral bone sizes was another concern that we investigate. In univariate analysis, smaller distal femoral bone and larger prosthesis compared to bone or PDFW ratio were the risk factors of PFF. However, these factors were not significant when fitting to the regression model. For femoral component positioning, the correlation between malalignment and PFF was not well documented. Although LDFA in PFF group was significantly higher than the control group, the amount of difference was not clinically important.

The most significant finding of our study existed that age and dyslipidemia were independent risk factors of early PFF. The cut-off point of age was $>=75$ years, while the odds ratio of dyslipidemia was 6.63 $(95 \% \mathrm{Cl}, 1.11$ to 39.8$)$. Several pieces of evidence about the effects of dyslipidemia on bone health had been proposed. Experimental studies showed that oxidized low-density lipoprotein (LDL) suppressed the differentiation of bone marrow stromal cells into osteoblasts and also promoted adipocytes.

Consequently, it might result in fatty marrow and bone mass reduction. Hypercholesterolemia could reduce bone mass via the inactivation of the LRP5/ 6 receptor and the involvement of Wnt signaling[10,11]. Pirie et al.[12] found that hyperlipidemia could induce secondary hyperparathyroidism, imparted bone regeneration, and mechanical strength in the animal model. In the cohort study, Poiana et al.[13] reported the significant correlation between bone mass density (BMD) and lipid profile in 
osteoporotic patients aged > 51 years. Furthermore, Bauer et al.[14] conducted a large meta-analysis of 151,500 patients and suggested that the use of statin medications for hyperlipidemia was associated with reduced fracture risk in an older woman. The association between dyslipidemia and PFF was not previously determined. As we have known, our study was a fundamental study that discovered this risk factor. However, our study had a small sample size, which made the wide $\mathrm{Cl}$ and did not collect the data about the statin user and level of lipid profile. Therefore, a larger well-controlled study was required to address this issue.

The most common mechanism for a supracondylar fracture was a low-velocity fall. Although AFN more than $3 \mathrm{~mm}$ with a sharp corner at the proximal end of a femoral component provided the highest stress concentration in a biomechanical study[15], a recent prospective clinical trial could not show the correlation between AFN and supracondylar fracture[16]. Our study also could not determine AFN as a risk factor. For the condylar fracture, Vestermark et al.[3]. found that seven patients sustained a condylar fracture in the acute postoperative setting. Five patients had preoperative valgus deformity and sustained fracture of unloaded medial condyle. The other two patients had preoperative varus deformity and sustained fracture of unloaded lateral condyle. The authors called this type of fracture as "early femoral condyle insufficiency fracture". Comparable to our study, all lateral condylar fractures had preoperative varus deformity. While $54.5 \%$ of medial condylar fractures had preoperative valgus deformity (Figure 3 ), we believed that insufficiency fracture might explain this phenomenon. Nevertheless, the remaining $45.5 \%$ of medial condylar fracture was still associated with preoperative varus deformity. Due to a fracture of the loaded medial condyle, it should be caused by a technical error during surgery.

In clinical application, appropriate surgical exposure, avoiding excessive bow cut if PS design was used, gentle trial reduction, and prosthesis insertion were essential for minimizing this complication. Because early PFF was not found in the CR design used, we recommended that the use of this design might be beneficial for high-risk patients. For surgeons who preferred PS design, intraoperative surveillance for occult fracture, and preparation of the backup femoral stem should be performed. Likewise, we thought that the prophylactic femoral stem insertion was another strategy to prevent PFF. A finite element study revealed that periprosthetic stress was reduced through the use of a femoral stem. It might help mitigate PFF risk[17]. However, big high-quality data was necessitated for stratifying or scoring the risk factors and identifying the appropriate patients.

There were several limitations to our study. First, our research was a retrospective design; retrieving some of the information that we need might be troublesome. Osteoporosis was one of the most critical factors that contribute to PFF risk. Bernatz et al.[18] reported that one-quarter of total joint arthroplasty patients met the criteria to receive osteoporosis medications. This lack of preoperative osteoporosis screening and treatment has also happened in our study. Second, our study's small sample size might decrease statistical power to detect the other significant risk factors. Third, most patients in this study were female that had a higher risk. Thus, our results could not be applied to male patients. Fourth, all radiographic outcomes were measured from short radiographs because we had not sent the full-length radiographs routinely in the early postoperative period. However, Alzahrani et al.[19] illustrated the good to the 
excellent correlation of short and full-length radiographs. They also suggested that short radiographs could be an appropriate substitute for full-length radiographs for evaluating postoperative coronal alignments. Lastly, although we tried to detect the consequent intraoperative PFF from immediate postoperative radiographs, it was challenging to distinguish the early PFF from occult intraoperative PFF.

In conclusion, we found that age and dyslipidemia were independent risk factors for early PFF. The cutoff point of age was $>=75$ years, with a sensitivity of $75.0 \%$ and specificity of $78.1 \%$. The odds ratio of dyslipidemia was $6.63(95 \% \mathrm{Cl}, 1.11$ to 39.8$)$. The further well-controlled studies with large sample size were needed to elucidate this research question. It would support us in doing strategic planning for preventing this complication.

\section{Declarations}

\section{Conflicts of interest}

All authors declare that there are no conflicts of interest in our study.

\section{Funding disclosure}

There is no funding support for this study.

\section{Acknowledgement}

The authors would like to thank Miss Nichakorn Khomawut for her assistance with data collection in this study.

\section{Compliance with Ethical standards}

- Ethical approval and consent to participate: The study was approved by the Institutional review board of Siriraj Hospital, Mahidol university.

- This study had been registered to Thaiclinicaltrial.gov (TCTR20200226001).

- Informed consent was obtained from all individual participants included in the study.

- Consent for publication: All authors have read and approved the final submitted manuscript.

- Availability of data and material: Requests for data not shown in the body of this manuscript can be made to the corresponding author

- Competing interests: The authors declare that they have no conflict of interest.

- Funding: There is no funding source.

- Authors' contributions: Chaturong Pornrattanamaneewong, M.D.,M.Sc and Keerati Chareancholvanich M.D. Akraporn Sitthitheerarut, M.D. provided research questions, conducted data collection, analyzed data, discussion and developed the full manuscript. Pakpoom Ruangsomboon 
M.D. examined all data analysis, detailed the results, statistical calculation and collected and monitored data. Rapeepat Narkbunnam M.D. also provided the research question and useful advice

- Acknowledgments: The authors acknowledge the assistance given by Nichakorn Khomawut with data collection. We also acknowledge Suthipol Udompunthurak, MSc.(Applied Statistics), for his assistance with the statistical analyses.

\section{References}

1. Zainul-Abidin S, Lim B, Bin-Abd-Razak HR, Gatot C, Allen JC, Koh J, et al. Periprosthetic Fractures after Total Knee Arthroplasty: the Influence of Pre-Operative Mechanical Factors versus Intraoperative Factors. Malays Orthop J. 2019 Jul;13(2):28-34.

2. Rorabeck CH, Taylor JW. PERIPROSTHETIC FRACTURES OF THE FEMUR COMPLICATING TOTAL KNEE ARTHROPLASTY. Orthop Clin North Am. 1999 Apr 1;30(2):265-77.

3. Vestermark GL, Odum SM, Springer BD. Early femoral condyle insufficiency fractures after total knee arthroplasty: treatment with delayed surgery and femoral component revision. Arthroplasty Today. 2018 Jun;4(2):249-53.

4. Backstein D, Safir O, Gross A. Periprosthetic Fractures of the Knee. J Arthroplasty. 2007 Jun 1;22(4, Supplement):45-9.

5. Wallace SS, Bechtold D, Sassoon A. Periprosthetic fractures of the distal femur after total knee arthroplasty : Plate versus nail fixation. Orthop Traumatol Surg Res OTSR. 2017;103(2):257-62.

6. Lim JBT, Bin Abd Razak HR, Zainul-Abidin S, Allen JC, Koh JSB, Howe TS. What Are the Preoperative Outcome Measures That Predispose to Periprosthetic Fractures After Primary Total Knee Arthroplasty? J Arthroplasty. 2017;32(8):2531-4.

7. Alden KJ, Duncan WH, Trousdale RT, Pagnano MW, Haidukewych GJ. Intraoperative Fracture During Primary Total Knee Arthroplasty. Clin Orthop Relat Res. 2009 May 9;468(1):90.

8. Lee JH, Wang S-I. Risk of Anterior Femoral Notching in Navigated Total Knee Arthroplasty. Clin Orthop Surg. 2015 Jun;7(2):217-24.

9. Culp RW, Schmidt RG, Hanks G, Mak A, Esterhai JL, Heppenstall RB. Supracondylar fracture of the femur following prosthetic knee arthroplasty. Clin Orthop. 1987 Sep;(222):212-22.

10. Yamaguchi T. [Bone metabolism in dyslipidemia and metabolic syndrome]. Clin Calcium. 2011 May;21(5):677-82.

11. Saarinen A, Saukkonen T, Kivelä T, Lahtinen U, Laine C, Somer M, et al. Low density lipoprotein receptor-related protein 5 (LRP5) mutations and osteoporosis, impaired glucose metabolism and hypercholesterolaemia. Clin Endocrinol (Oxf). 2010 Apr;72(4):481-8.

12. Pirih F, Lu J, Ye F, Bezouglaia O, Atti E, Ascenzi M, et al. Adverse Effects of Hyperlipidemia on Bone Regeneration and Strength. J Bone Miner Res. 2012 Feb;27(2):309-18.

13. Poiana C, Radoi V, Carsote M, Bilezikian JP. New Clues that May Link Osteoporosis to the Circulating Lipid Profile. Bone Res. 2013 Sep;1(3):260-6. 
14. Bauer DC, Mundy GR, Jamal SA, Black DM, Cauley JA, Ensrud KE, et al. Use of statins and fracture: results of 4 prospective studies and cumulative meta-analysis of observational studies and controlled trials. Arch Intern Med. 2004 Jan 26;164(2):146-52.

15. Zalzal P, Backstein D, Gross AE, Papini M. Notching of the anterior femoral cortex during total knee arthroplasty characteristics that increase local stresses. J Arthroplasty. 2006 Aug;21(5):737-43.

16. Puranik HG, Mukartihal R, Patil SS, Dhanasekaran SR, Menon VK. Does Femoral Notching During Total Knee Arthroplasty Influence Periprosthetic Fracture. A Prospective Study. J Arthroplasty. 2019;34(6):1244-9.

17. Conlisk N, Howie CR, Pankaj P. Optimum stem length for mitigation of periprosthetic fracture risk following primary total knee arthroplasty: a finite element study. Knee Surg Sports Traumatol Arthrosc Off J ESSKA. 2018 May;26(5):1420-8.

18. Bernatz JT, Brooks AE, Squire MW, Illgen RI, Binkley NC, Anderson PA. Osteoporosis Is Common and Undertreated Prior to Total Joint Arthroplasty. J Arthroplasty. 2019;34(7):1347-53.

19. Alzahrani MM, Wood TJ, Somerville LE, Howard JL, Lanting BA, Vasarhelyi EM. Correlation of Short Knee Radiographs and Full-length Radiographs in Patients Undergoing Total Knee Arthroplasty. J Am Acad Orthop Surg. 2019 Jun 1;27(11):e516-21.

\section{Tables}

Table 1. Patients' characteristics 


\begin{tabular}{|c|c|c|c|}
\hline Characteristics & PFF group $(\mathrm{n}=24)$ & Control group $(n=96)$ & $p$-value \\
\hline Age (yr) & $77.1 \pm 6.7$ & $69.0 \pm 7.2$ & $<0.001^{*}$ \\
\hline Gender (female,\%) & $22(91.7 \%)$ & $84(87.5 \%)$ & 0.733 \\
\hline Weight (kg) & $63.5 \pm 11.2$ & $66.5 \pm 12.8$ & 0.287 \\
\hline Height (cm) & $152.6 \pm 7.6$ & $154.9 \pm 7.8$ & 0.205 \\
\hline $\mathrm{BMI}\left(\mathrm{kg} / \mathrm{m}^{2}\right)$ & $27.3 \pm 4.6$ & $27.7 \pm 4.8$ & 0.701 \\
\hline Side (right,\%) & 20 (83.3\%) & $53(55.2 \%)$ & $0.012^{\star}$ \\
\hline DCCl (scores) & $0.5 \pm 0.9$ & $0.3 \pm 0.5$ & 0.348 \\
\hline \multicolumn{4}{|l|}{ Diagnosis (\%) } \\
\hline Osteoarthritis & $22(91.7 \%)$ & $96(100.0 \%)$ & $0.039 *$ \\
\hline Rheumatoid arthritis & $2(8.3 \%)$ & $0(0.0 \%)$ & \\
\hline \multicolumn{4}{|l|}{ Underlying diseases (\%) } \\
\hline Diabetes & $5(20.8 \%)$ & $25(26.0 \%)$ & 0.598 \\
\hline Hypertension & $18(75.0 \%)$ & $73(76.0 \%)$ & 0.915 \\
\hline Dyslipidemia & $14(58.3 \%)$ & $34(35.4 \%)$ & $0.040^{\star}$ \\
\hline Cardiovascular disease & $2(8.3 \%)$ & $5(5.2 \%)$ & 0.626 \\
\hline Thyroid disease & $1(4.2 \%)$ & $1(1.0 \%)$ & 0.361 \\
\hline Parkinson disease & $3(12.5 \%)$ & $0(0.0 \%)$ & $0.007 *$ \\
\hline Steroid use (\%) & $1(4.2 \%)$ & $0(0.0 \%)$ & 0.200 \\
\hline \multicolumn{4}{|l|}{ Surgical approach (\%) } \\
\hline Medial parapatellar & $23(95.8 \%)$ & $95(99.0 \%)$ & 0.361 \\
\hline Midvastus & $1(4.2 \%)$ & $1(1.0 \%)$ & \\
\hline Operative time $(\mathrm{min})$ & $81.5 \pm 23.4$ & $79.9 \pm 30.2$ & 0.807 \\
\hline \multicolumn{4}{|l|}{ Prosthesis design (\%) } \\
\hline Cruciate-retaining & $0(0.0 \%)$ & $24(25.0 \%)$ & $0.004^{\star}$ \\
\hline Posterior-stabilized & $24(100.0 \%)$ & $72(75.0 \%)$ & \\
\hline \multicolumn{4}{|l|}{ Prosthesis brand (\%) } \\
\hline Zimmer & 20 (83.3\%) & $63(65.6 \%)$ & $0.046^{\star}$ \\
\hline Depuy & $3(12.5 \%)$ & 31 (32.3\%) & \\
\hline
\end{tabular}




\begin{tabular}{|lll|} 
Stryker & $1(4.2 \%)$ & $0(0.0 \%)$ \\
Smith Nephew & $0(0.0 \%)$ & $2(2.1 \%)$ \\
\hline
\end{tabular}

PFF, periprosthetic femoral fracture; BMI, body mass index; DCCl, Deyo-Charlson comorbidity index *p-value $<0.05$

Table 2. Radiographic outcomes

\begin{tabular}{|llll|}
\hline Outcomes & PFF group $(\mathrm{n}=24)$ & Control group $(\mathrm{n}=96)$ & -value \\
\hline DFW $(\mathrm{mm})$ & $80.4 \pm 5.3$ & $85.7 \pm 7.0$ & $0.001^{*}$ \\
\hline PDFW ratio & $0.79 \pm 0.01$ & $0.75 \pm 0.05$ & $0.004^{*}$ \\
\hline LDFA $\left(^{\circ}\right)$ & $86.2 \pm 2.0$ & $85.2 \pm 1.9$ & $0.026^{*}$ \\
\hline FCFA $\left({ }^{\circ}\right)$ & $88.4 \pm 6.0$ & $87.8 \pm 4.1$ & 0.635 \\
\hline AFN $(\%)$ & $2(8.3 \%)$ & $1(1.0 \%)$ & 0.102 \\
\hline AFN $(\mathrm{mm})$ & $0.3 \pm 1.1$ & $0.1 \pm 0.0$ & 0.301 \\
\hline
\end{tabular}

PFF, periprosthetic femoral fracture; DFW, distal femoral width; PDFW, prosthesis-distal femoral width; LDFA, anatomical lateral distal femoral angle; FCFA, femoral component flexion angle; AFN, anterior femoral notching

*p-value $<0.05$

Table 3. Patterns and data of periprosthetic fracture 


\begin{tabular}{|lllll|}
\hline Data & $\begin{array}{l}\text { Medial condylar } \\
\text { fracture }\end{array}$ & $\begin{array}{l}\text { Lateral condylar } \\
\text { fracture }\end{array}$ & $\begin{array}{l}\text { Supracondylar } \\
\text { fracture }\end{array}$ & $\begin{array}{c}\text { p- } \\
\text { value }\end{array}$ \\
\hline Number $(\%)$ & $11(45.8 \%)$ & $6(25.0 \%)$ & $7(29.2 \%)$ & NA \\
\hline $\begin{array}{l}\text { Preoperative } \\
\text { deformity }(\%)\end{array}$ & & & & \\
\hline -Varus & $5(45.5 \%)$ & $6(100.0 \%)$ & $6(85.7 \%)$ & $0.036^{*}$ \\
\hline -Valgus & $6(54.5 \%)$ & $0(0.0 \%)$ & $1(14.3 \%)$ & \\
\hline \begin{tabular}{l} 
aFTA $\left(^{\circ}\right)$ \\
\hline $\begin{array}{l}\text { Time to fracture } \\
\text { (days) }\end{array}$
\end{tabular} & $176.3 \pm 10.3$ & $189.8 \pm 5.3$ & $183.3 \pm 11.5$ & $0.036^{*}$ \\
\hline
\end{tabular}

aFTA, anatomical femorotibial angle, NA, not applicable

*p-value $<0.05$

Figures

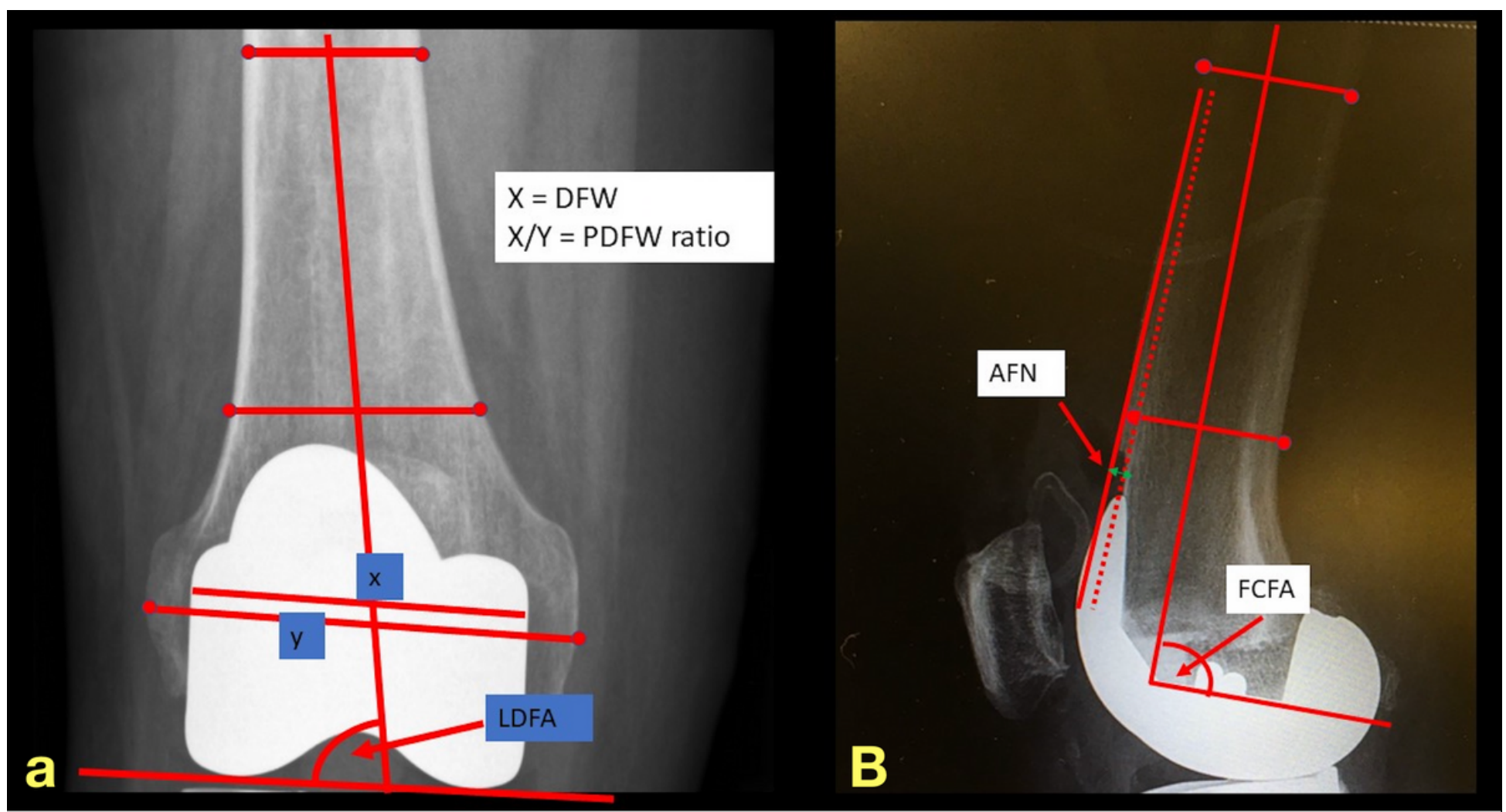

Figure 1 
The measurements of knee radiographic outcomes from a) anteroposterior view (anatomical lateral distal femoral angle, LDFA; distal femoral width, DFW; and prosthesis-distal femoral width ratio, PDFW) and b) lateral view (femoral component flexion angle, FCFA; and anterior femoral notching, AFN)

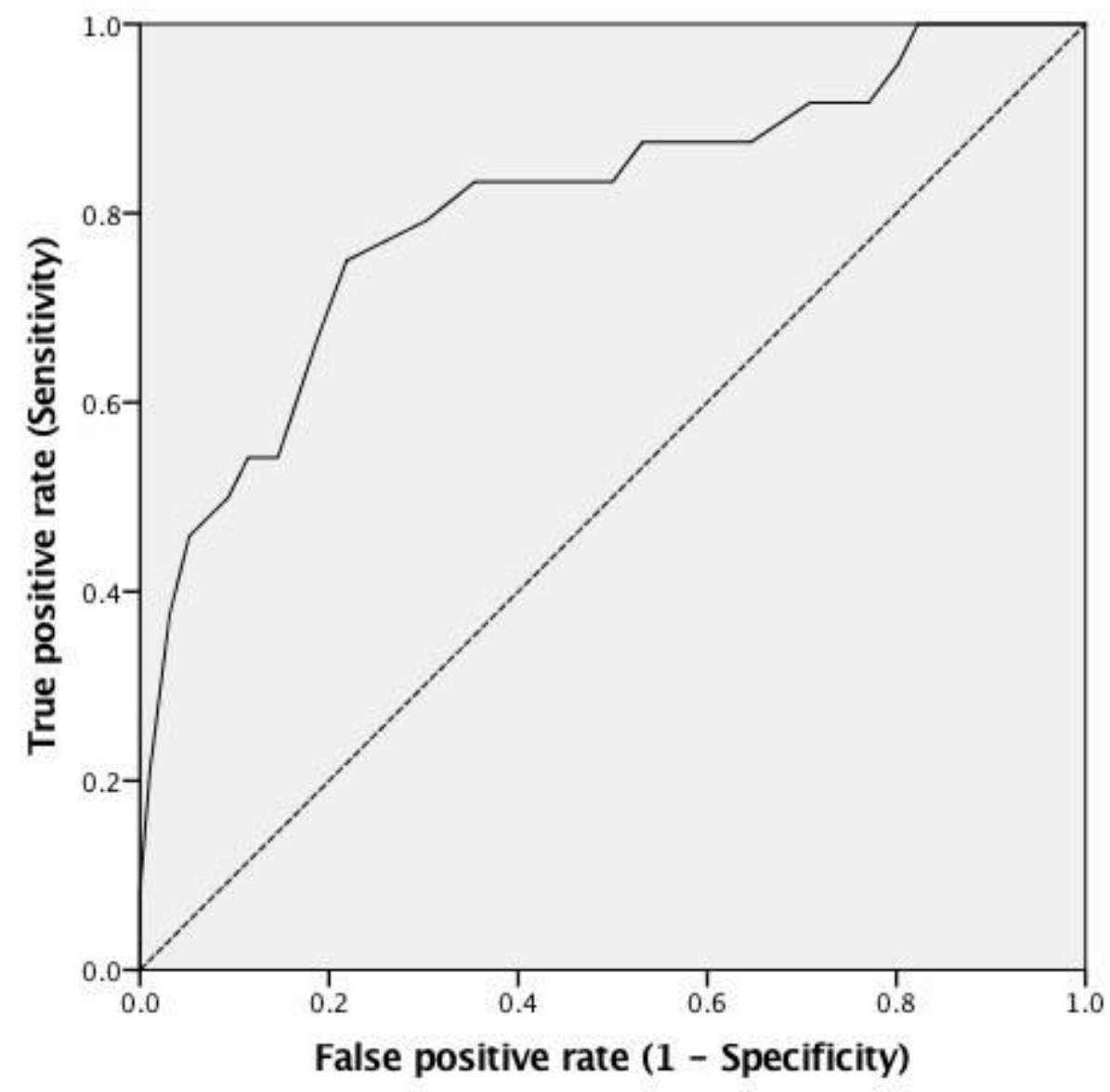

Figure 2

The receiver operating characteristics curve for prediction of early periprosthetic femoral fracture based on the age at index of total knee arthroplasty 


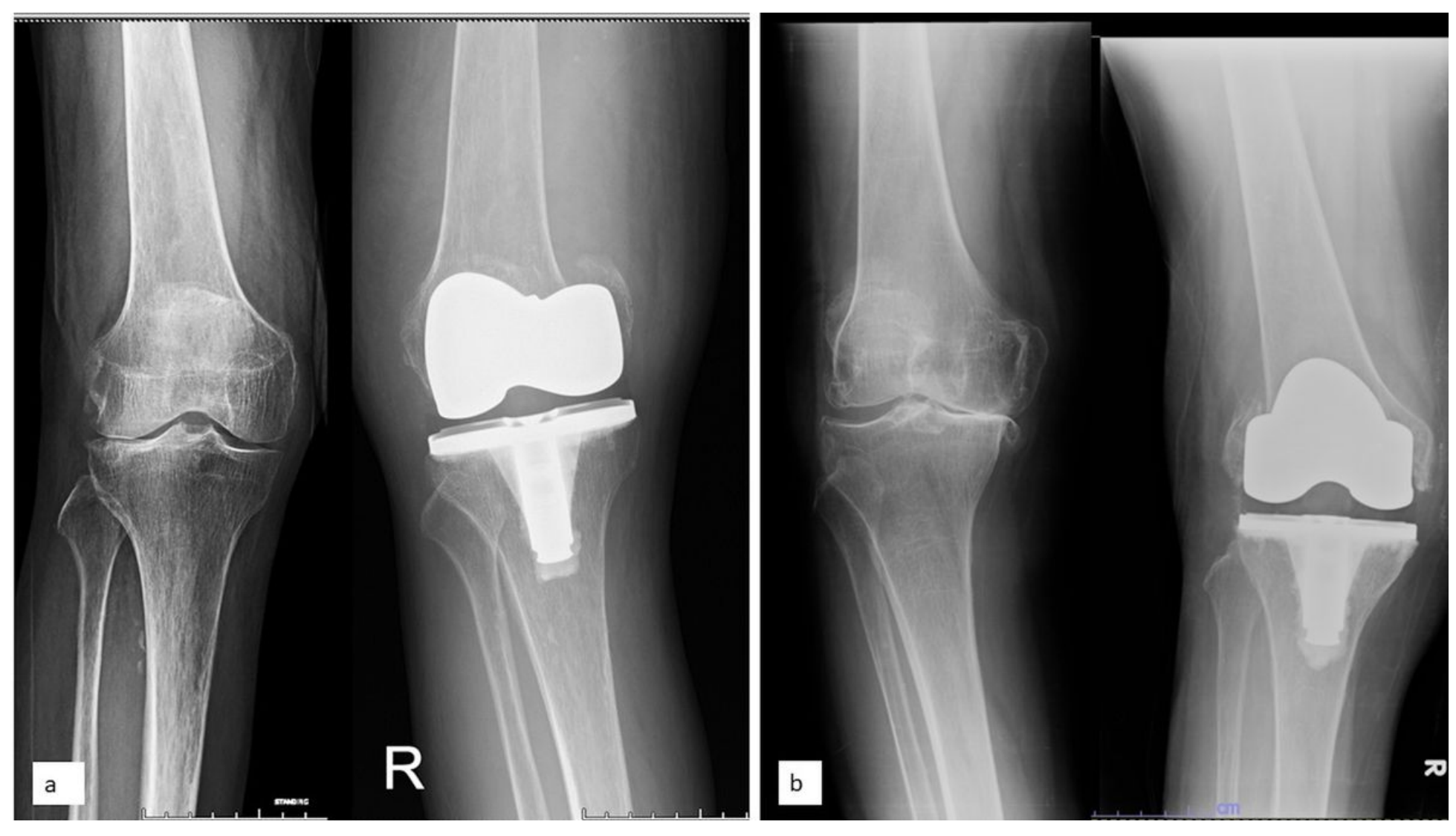

Figure 3

Preoperative and postoperative anteroposterior knee radiographs of two patients demonstrating a) medial condylar fracture and $b$ ) lateral condylar fracture, respectively 